\title{
Overnight fasting affects avoidance learning and relief
}

\author{
Silvia Papalini ${ }^{1,2, \bigotimes}$, Laura Neefs ${ }^{1}$, Tom Beckers ${ }^{2,5}$, Lukas Van Oudenhove ${ }^{2,3,4}$, and Bram Vervliet ${ }^{1,2}$ \\ ${ }^{1}$ Laboratory of Biological Psychology, Faculty of Psychology and Educational Sciences, KU Leuven, Belgium. \\ ${ }^{2}$ Leuven Brain Institute, KU Leuven, Belgium. \\ ${ }^{3}$ Laboratory for Brain-Gut Axis Studies, Translational Research Center for Gastrointestinal Disorders, Department of Chronic Diseases and Metabolism, KU \\ Leuven, Belgium. \\ ${ }^{4}$ Cognitive and Affective Neuroscience Lab, Department of Psychological and Brain Sciences, Dartmouth College, Hanover, NH, USA. \\ ${ }^{5}$ Centre for the Psychology of Learning and Experimental Psychopathology, Faculty of Psychology and Educational Sciences, KU Leuven, Belgium.
}

\begin{abstract}
Prolonged fasting influences threat and reward processing, two fundamental systems underpinning adaptive behaviors. In animals, overnight fasting sensitizes the mesolimbic-dopaminergic activity governing avoidance, reward, and fear-extinction learning. Despite evidence that overnight fasting may also affect reward and fear learning in humans, effects on human avoidance learning have not been studied yet. Here, we examined the effects of 16h-overnight fasting on instrumental avoidance and relief from threat omission. To this end, 50 healthy women were randomly assigned to a fasting $(\mathrm{N}=25)$ or a re-feeding group $(\mathrm{N}=25)$ and performed an Avoidance-Relief Task. We found that fasting decreases unnecessary avoidance during signaled safety; this effect was mediated via a reduction in relief pleasantness during signaled absence of threat. A fasting-induced reduction in relief was also found during fear extinction learning. We conclude that fasting optimizes avoidance and safety learning. Future studies should test whether these effects also hold for anxious individuals.
\end{abstract}

Keywords: fasting, food deprivation, avoidance, extinction, reward, relief, anxiety

Correspondence: silvia.papalini@kuleuven.be

\section{Statement of relevance}

Threat omission induces relief, a pleasant feeling that arises from a better than expected outcome. Relief pleasantness reinforces avoidance behavior. Hence, exaggerated relief pleasantness might contribute to excessive avoidance, the typical hallmark of anxiety, and the behavior that prevents the instauration of new 'safer' learning. Relief and avoidance learning largely depend upon the activation of the mesolimbic-dopaminergic system. Hence, interventions with a dopaminergic effect can help to understand the nature of excessive avoidance and its treatment. Since hunger increases dopamine-reward sensitivity, we adopted fasting as a procedure that might indirectly increase dopamine activation during relief and avoidance. Unexpectedly, in the hungry group, a reduction in relief pleasantness during the absence of threat fully mediated a reduction in unnecessary avoidance to a fared stimulus. Hence, a simple diet manipulation with a cleverly designed intervention might optimize the learning processes that take part into excessive avoidance and its treatment.

\section{Introduction}

Avoidance consists of actions that individuals adopt to guarantee self-protection from expected threats[5, 15]. However, when threat expectations are unrealistic (e.g., in anxiety disorders), avoidance can become inflexible, repetitive, and maladaptive[2]. Excessive avoidance limits new experiences, such as when social events or job opportunities, are continually avoided, leading to a drastic reduction in subjective well-being[23]. Consequently, a major goal of clinical interventions for anxiety disorders is to decrease excessive avoidance, often by reducing elevated fear. However, the relation between excessive avoidance and elevated fear is complicated, and experimental/clinical studies showed that avoidance can endure without the presence of fear[7, 42]. Given the relatively fear-independent and repetitive nature of excessive avoidance, research on fear conditioning and its extinction is integrating reward-related processes to explain how avoidance behaviors are maintained[26, 32, 43, 45]. Specifically, instrumental avoidance learning has been shown to rely on the same dopaminergic neurotransmitter system that is involved in reward learning[28]. 
In fact, following fear acquisition, when an action toward a warning signal activates a safety signal and can subsequently entirely prevent a threat, an increased release of midbrain dopamine is observed[24]. This indicates that, upon continued presentations of such warning and safety signals, striatal dopaminergic neurons acquire predictive responses to threat omissions. Such property underpins the acquisition of conditioned avoidance learning[24]. Hence, manipulating DA-related activity during threat omissions should affect instrumental avoidance learning and its maintenance. Similarly, fear extinction learning relies upon dopamine-based reward prediction error (PE), specifically at the level of the ventral tegmental area (VTA)[5, 15]. Recent optogenetic studies in animals show that disruption (or enhancement) of activity of dopaminergic neurons in the VTA during unexpected threat omissions decreases (or enhances) fear extinction learning[18, 31]. Fear extinction is the progressive decay of a conditional response following the repeated presentations of the conditional stimulus (CS) in absence of the paired threat (unconditional stimulus, US)[41]. Of note, exposure-based therapies rely in part on fear extinction learning[27]. During such therapy, anxious individuals are repeatedly exposed to the fear-eliciting situation within a safe context. This constitutes a violation of the erroneous expectation of threat and leads to a reduction of fear. Each exposure triggers a betterthan-expected outcome (reward-PE), the magnitude of which will depend on the magnitude of the threat expected by the individual[27]. Therefore, variations in DA-related activity might also modulate PE during threat omissions, and consequently, influence fear extinction learning.

It is well known that prolonged fasting exerts strong influences on the mesolimbic DA-system in the brain, in the service of increasing reward-related emotions and motivations[6, 9, 12, 21, 30, 36, 44, 46]. Particularly, prolonged fasting increases the reinforcing properties of stimuli that lead to a phasic release of dopamine[6]. Indeed, food restriction increases food-approach behaviors and exacerbates symptoms in eating disorders (e.g. binge eating)[25]. In animals, fasting influences the balance between threat avoidance and reward-seeking, since prey animals usually forage for food in territories with potential threat of predators. The current literature on fasting and fear instead focuses mainly on fear extinction. Specifically, animal studies demonstrate that overnight fasting increases extinction learning[40], promotes extinction recall[40], and decreases long-term fear memory[40]. These effects are mediated by increased levels in plasma acylated ghrelin[13], a gastric hormone regulating energy balance and the dopaminergic reward system[10]. Although animal studies highlight a role for overnight fasting in modulating fear and dopaminebased reward mechanisms, no evidence of a link between fasting, the reward, and the fear system exists in the literature on avoidance learning in humans.

In healthy humans, overnight fasting also affects both reward and fear-related mechanisms. Within the context of fasting and reward, recent studies in humans indicated that fasting increased incentive salience at the level of the meso-cortico-limbic DA-system[46], and delay discounting for non-food rewards[36]. This is consistent with the evidence that prolonged fasting increases the hedonic value not only of food (primary reward)[22, 38], but also of other types of rewards (such as money), by enhancing general DA-based reward sensitivity[9]. Within the context of fasting and fear, le Shi and colleagues[35] found that overnight fasting increased fear extinction retention, and reduced fear renewal. Furthermore, similarly to animals, higher plasma levels of ghrelin were associated with reduced fear at test[35]. Consequently, fasting might increase general PE-based reinforcement learning, hence also within the context of instrumental avoidance and fear extinction learning (e.g., by enhancing the reward value of threat omission, i.e., relief pleasantness). To date, however, neither animal nor human studies investigated the effect of overnight fasting on avoidance and PE during threat omission. Here, we examine the consequences of overnight fasting on these processes by using a specific task- based proxy for the dopamine-based reward PE. Omissions of an expected aversive event trigger a pleasant feeling of surprise that can be measured as subjective relief[45] and correlates with Nucleus accumbens (Nacc)[16] activation, one of the main brain areas involved in reward processing and PE. Relief pleasantness is particularly elevated when an upcoming threat is omitted by an avoidance action, and gradually decreases over repeated actions as the omission of the threat becomes less surprising[32, 43]. For these reasons, relief pleasantness serves as a proxy to the hedonic reaction to a dopamine-based reward PE during instrumental avoidance learning. Therefore, for the purpose of this study, we use relief pleasantness as a PE-related index to investigate the potential effects of overnight fasting on the DA-system within the context of instrumental avoidance and fear extinction learning.

We examined 1) whether overnight fasting increases instrumental avoidance learning, and 2) whether overnight fasting increases fear extinction learning. We then investigated 3) whether the effect of fasting on instrumental avoidance learning is mediated by its effects on relief pleasantness. Finally, we investigated whether 4a) fasting increases the return of avoidance (as a form of persistency) after fear extinction, and $4 \mathrm{~b}$ ) whether this fasting- induced persistence of avoidance after extinction is mediated by the effects of fasting on relief pleasantness (preregistration: https://osf.io/yqp3x/). 


\section{Methods}

\section{Participants.}

Participants were fifty healthy participants from the Leuven area (age: 22.26/3.76, Body Mass Index [BMI]: 21.10/1.79, Mean/SD). Written informed consent was obtained from all participants and in agreement with the local Social and Medical Ethics Committee (SMEC, Leuven, Belgium). We only included Dutch-speaking females taking hormonal contraceptives to keep as much control as possible on hormone levels. Participants enrolled themselves in the study in return for 2.5-course credits or a financial reward of 20 Euros per hour, plus a free breakfast. Criteria of exclusion were decided on the basis of the nutritional-based nature of the study and the methodology used (i.e. electric stimulation). The list of the criteria can be found in the Supplementary Material.

Sample size and group assignment To ensure sufficient statistical power for our analyses, fifty healthy volunteers were

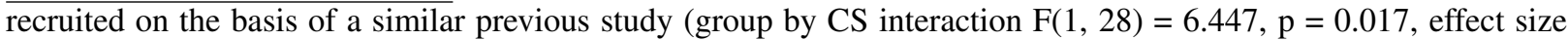
f: 0.4636) on the effects of hunger on return of fear (Le Shi et al., 2018, experiment 3). Given that our hypotheses regarded mostly the Group-by-CS interaction the power calculation was performed including a number of 2 Group and 3 CSs. The $\mathrm{g}^{*}$ power calculation indicated a 20 total sample size for a power of $95 \%$ and alpha of .001, which was adjusted to 50 to further increase power, as pre-registered. Participants were randomly assigned to the Re-feeding $(\mathrm{N}=25)$ or to the Fasting group $(\mathrm{N}=25)$.

\section{Procedures.}

Fasting Manipulation The study consisted of two visits. During a screening visit (25 minutes) participants were informed about the nature of the study and procedures. Afterwards, they were randomly assigned to the Re-feeding or to the Fasting Group. Participants were instructed on dietary requirements (including no intake of soft drinks) and we provided a diary to keep track of food intake, medication use, and sleep quality of the night preceding the testing session. The evening before the testing session, participants were additionally asked to keep physical activity at a minimum. On the day preceding the testing session, participants were asked to consume a regular size dinner and to start fasting at 6PM. The testing session started at 8AM of the next day (125 minutes). Participants from the Re-feeding Group received breakfast while the Fasting Group got access to food only at the end of the experiment (at the latest at 10:00AM), see Figure 1. A standard breakfast consisted of two slices of (toast size) bread with jam/cheese, one apple, one regular glass of milk or juice, and one full-fat yoghurt. Before the start of the behavioral task the glucose, hunger, and ketone levels were measured. Next, participants from the Re-feeding Group consumed a standard breakfast (see Supplementary Material) (10 minutes) and read a magazine (15 minutes) before the start of the task while participants from the Fasting Group were left free to read the same material for about 25 minutes. After a second glucose and hunger measurement, the patches for the skin conductance and the bracelet for the electrical stimulation (US) were applied. The US intensity was determined by the participant through a work-up procedure before the start of behavioral task.

\section{Stimuli and apparatus.}

Stimuli Stimuli were presented in Affect 5[37]. Two different backgrounds were presented on a computer screen (an office room and a conference room), serving as contexts. Both backgrounds contained a desk lamp that could light-up in three different colors: red, blue, and yellow. The three different colors of the lamp served as conditional stimuli (CSs). The unconditional stimulus (US) was electrical stimulation. There were three types of conditional stimuli: (1) the CS-, (2) the CS+ avoidable $\left(\mathrm{CS}+_{\text {avoidable }}\right)$ and (3) the $\mathrm{CS}+$ unavoidable $\left(\mathrm{CS}+{ }_{\text {unavoidable }}\right)$. The stimuli were counterbalanced and a CS never appeared more than two times after another in all experimental phases. The CSs+ were followed by an electrical stimulation (100\% reinforcement) and the CS- was not. Avoidance action could

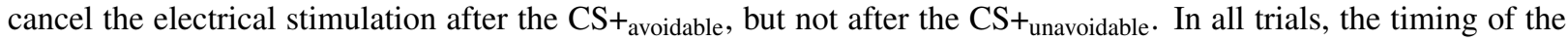
stimulus presentation was jittered to avoid learning effects. Each trial started with the context presentation, i.e. with a non-lit lamp (2-3s). After this, the lamp was lit with one of the three colors (CS) (7.5-9s). Next, a black screen was presented (4.5-6s). Then, the relief rating appeared for $6 \mathrm{~s}$ or until participants confirmed their rating with the left mouse button. Intertrial intervals varied between 12 and 15.5 seconds, with an average of 15 seconds.

To deliver the electric stimulation, a DS7 device was used (Digitimer@, Hertfordshire, UK). This stimulation consisted of high-voltage brief 2-ms electrical pulses. The pulse was delivered by two adjacent SensorMedics 


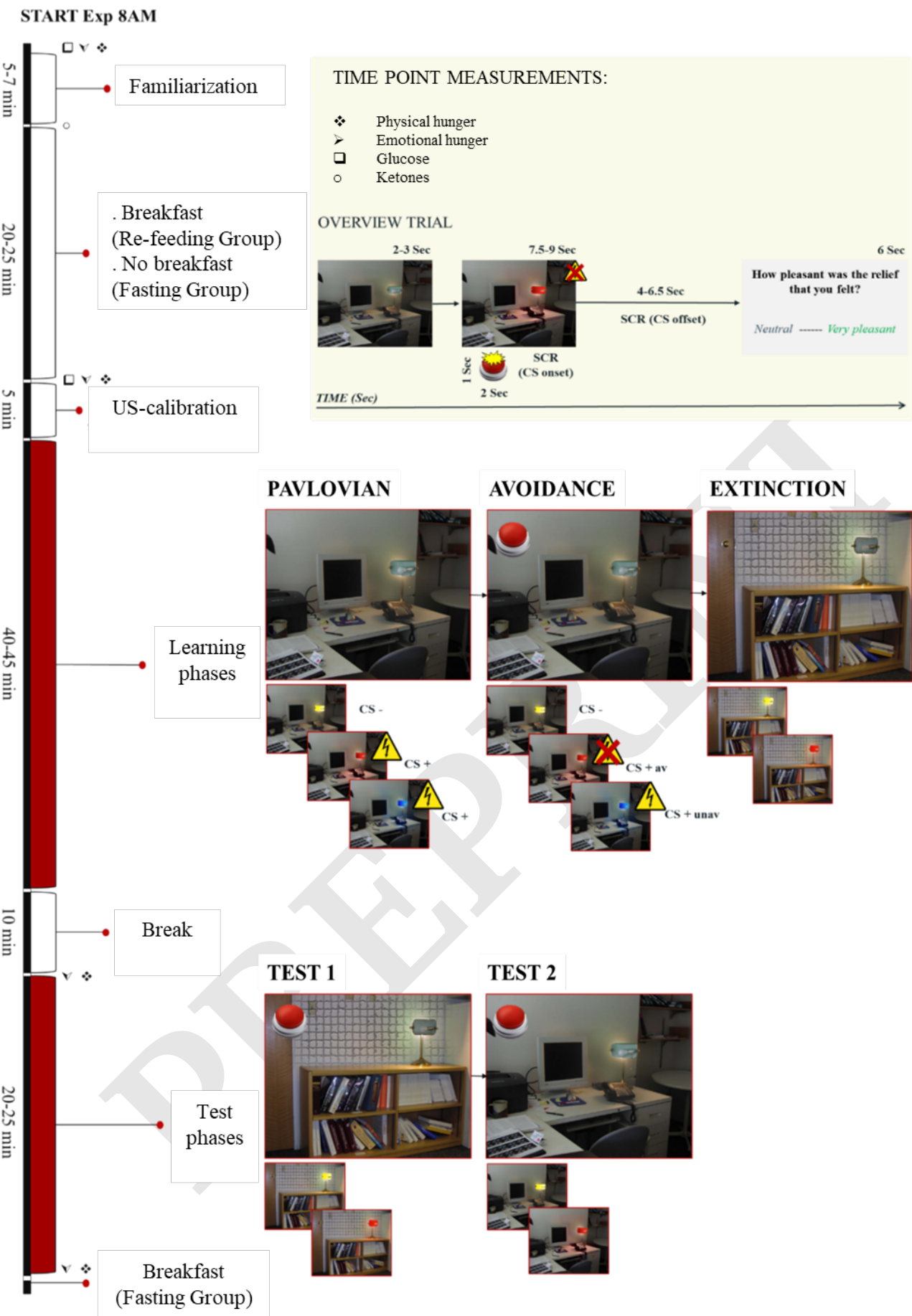

END $\operatorname{Exp} 10 \mathrm{AM}$

Figure 1. Overview of the Avoidance and Relief Task (ART) and the procedures applied during the testing session. After having measured their hunger and ketone and glucose levels, half of the participants were asked to consume a standard breakfast. All participants then performed the ART task. The task included a Pavlovian, avoidance, and fear extinction learning phase, followed by two test phases to measure persistent avoidance. The effects of fasting vs re-feeding are tested on different measurements recorded during the various phases of the task, such as the amount of button presses (avoidance actions) and individual relief pleasantness ratings collected each time the electrical stimulation was omitted (see methods section for a full explanation). 
surface-electrodes, filled with KY gel. These electrodes were placed at the forearm of the non-dominant hand of the participant. The level of the electrical stimulation was selected by the participant before the start of the task via a gradual intensifying procedure ranging from 0 to 5 . The level that was 'highly annoying, but not painful', level 5, was used for the whole ART.

Physiology Ketone levels were measured using Ketostiks strips (Ascensia Diabetes Care), while glucose levels were assessed via a standard glucometer device (GlucoMen@areo Sensor). Skin conductance levels (SCL) were measured via a Coulbourn LabLinc V equipment. This equipment was linked to two sensors which were attached to the palm of the non-dominant hand of the participant.

Self-reported rating Physical ('how hungry are you?') and emotional ('how strong is your desire to eat?') hunger was measured using a visual analogue scale (VAS) appearing on the computer screen. US-expectancy was measured retrospectively on a Likert scale ranging from 1 (shock not expected) to 10 (shock expected). The pleasantness of relief was measured on a Likert scale ranging from 1 (neutral) to 4 (very pleasant). This scale appeared on the computer screen every time the US was not delivered.

Behavioural outcome Avoidance actions were measured in terms of button clicks.

\section{The Avoidance-Relief Task (ART).}

Each participant performed the Avoidance and Relief Task. The ART consists of three learning phases and two tests: (1) Pavlovian conditioning, (2) avoidance conditioning, (3) fear extinction learning, (4) extinction recall (5) and fear renewal (Figure 1).

Participants were first exposed to the visual stimuli during a familiarization phase. Subsequently, during the Pavlovian learning phase (16 trials), they were instructed to learn the association between the CS and the electrical stimulation (US). During this phase, two lamp colors (CS+) are followed by the US, while the third (CS-) is not. During this phase, all the stimuli are presented in context A. Next, during the Avoidance conditioning phase (36 trials), the possibility to avoid the US is introduced by the presentation of a red button on the screen. Participants are instructed that pressing the button might or might not cancel the delivery of the shock. Clicking the button is effective to cancel the US during one CS+ $\left(\mathrm{CS}+{ }_{\text {avoidable }}\right)$, but not to the other $\mathrm{CS}+\left(\mathrm{CS}+_{\text {unavoidable }}\right)$, while it is unnecessary during the safe CS-.

During the subsequent Extinction phase (16 trials), the $\mathrm{CS}+_{\text {avoidable }}$ and the CS- are presented on a different background (extinction room/context B), in absence of the button and the electric stimulation. After a 10 minute break, Test 1 ( 8 trials) starts by reintroducing the red button in the extinction context B. During this phase, no US is delivered. Finally, Test 2 ( 8 trials) is identical to the extinction phase with the difference that the CSs are presented in the conditioning context A. Of note, at the beginning of each phase, participants are instructed that they might or might not receive the electrical stimulation.

\section{Analysis}

The statistical analyses were conducted by a researcher (SP) who was blind to the code assigned to the groups (Refeeding or Fasting group) to eliminate unintended bias of the results. De-blinding was applied only after the central pre-registered hypotheses were addressed and all the group comparisons performed. All participants were included in the analysis since differential avoidance (number of button press for the $\mathrm{CS}+{ }_{\text {avoidable }}>$ number of button press for the $\mathrm{CS}_{\text {unavoidable }}$ and for the CS-), the main outcome of the paradigm, was successfully acquired by each individual over the course of the trials of the paradigm.

Differently from the pre-registration, the button press, relief ratings, SCR (during US anticipation and CS offset), and retrospective US expectancy ratings were analyzed as dependent variables in a consistent manner via (generalized) linear mixed models (GLMM and LMM)[20] in SPSS (IBM Corp. Released 2020. IBM SPSS Statistics for Windows, Version 27.0. Armonk, NY). This choice was based on the presence of binomial (button press) and multinomial (relief ratings) distributions of the data, missing data for the relief scores, and absence of normal distribution of the continuous data. The normality of the distribution of the residuals was visually checked within each LMM 
model. The independent variables were 'Group', type of 'CS', and 'Trial'. 'Trial' was used as a categorical dichotomous variable for the analysis of the retrospective expectancy ratings [first and last presentation of each CS], and as a continuous de-meaned variable for the remaining analysis. In all the models we included subject as a random intercept, but we did not include a random slope since this term did not improve the models fit based on the Bayesian Information Criterion (BIC). Also, in each model the CS- (CS $\rightarrow$ noUS) was taken as reference category to check the presence of differential learning so that the CS+ (CS $\rightarrow$ US) vs CS- (CS $\rightarrow$ noUS) served as a manipulation check. Additionally, for the analysis relative to the SCR during the avoidance phase we included the avoidance action (yes or no) as a covariate (only main effect). Results from the full models including the contrasts that served as a manipulation check are reported in the Supplementary Material. In the main text, we only report the principal contrasts of interest to test our pre-registered hypotheses, which include the main effect of Group (Fasting vs Re-feeding), Group by CS (CS+ vs CS-), and Group by CS (CS+ vs CS-) by Trial interaction effects during the Avoidance and Extinction phase. For the Test phases we did not include the Group by CS (CS+ vs CS-) by Trial interaction given the few trials included in these phases. Significant p-values were considered when below an alpha- value of 0.05. Omnibus F-tests as well as regression $\beta$ weights (together with the standard errors, $\pm \mathrm{SE}$ ) were reported for the significant fixed main and interaction effects testing the hypotheses. Post hoc LMM or GLMM analyses run separately for each CS type were used only to further characterize significant interactions. A correction for a factor of 3 or 2 was applied to the alpha-value of the results from the Post hoc model.

Skin conductance data were filtered using a bandpass filter[3] and analyzed in MATLAB R2018b to calculate the skin conductance response (SCR) for the onset (US- anticipation) and offset (US-omission) of the CS. For the US-anticipation, the SCR was calculated by subtracting the average SCL during the 1 second prior to appearance of the CS on the screen, to the peak found during the presentation of the CS $(7.5-9 \mathrm{sec})$. The SCR to the omission of the US was calculated by subtracting the average SCL during 1 second before CS offset from the peak found between CS offset and start of the relief rating $(4.5-6 \mathrm{sec})$. A square root transformation was applied to each value to reduce the skewness of the distribution since values $<.002$ were set to 0 (values lower than .002 are communally not considered as a response to the stimulus in fear conditioning paradigms). For SCRs which transformation did not resulted in a normal distribution, we run a mixed-effects mixed distribution model for zero-inflated data[39]. Specifically, for this analysis, a division between the zero-inflated SCR distribution in values equal or greater to zero was made. Subsequently, for the logistic component, we ran a GLMM for binary data estimating the conditional probability of the occurrence of SCR values above zero (which served as an index of the amount of the responders to the CSs). For the lognormal component, a LMM was run using the normalized non-zero values of the SCR (which served as a measure of magnitude of the skin response to the CSs). In both the components we included a random subject factor and the three fixed effects. For the GLMM models, the Odds Ratio $(\exp -\beta)$ served as a measure of Effect Size. For the LMM models, we reported the Effect Size (d [small: .20; medium: .50; large: .80]) for the main effect of Group dividing the difference of the means from the two groups by the square root of the variance of the residuals plus the variance of the intercept (random subjects)[8].

Finally, for the mediation analysis (simple and serial), a Bootstrapping method (95\% of the indirect effect obtained with 5000 bootstrap resample) was used. For the mediation analysis we only used the avoidance actions relative to

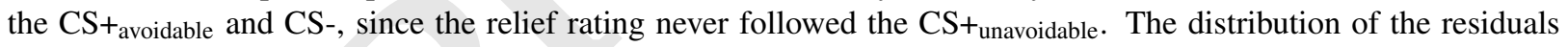
of the mediators (differential relief and differential avoidance) were normally distributed, and so no correction was applied.

\section{Results}

Results from the fasting manipulation on glucose and hunger levels, as well as the complete results from the manipulations during the different learning phases of the task (US/noUS- related manipulation check), can be found in the Supplementary Material; they are in line with expectations. No analyses were performed for the ketone levels since non-zero values were found in three participants only.

\section{Avoidance learning: overnight fasting decreases avoidance frequency and relief pleasantness (hypoth- esis 1).}

Avoidance action frequency: The 3 (CS: CS-, CS+ $+_{\text {avoidable }}, \mathrm{CS}+_{\text {unavoidable }}$ ) by 2 (Group: Fasting, Re-feeding) by Trial (continuous variable) GLMM analysis revealed a main effect of $\operatorname{Group}(F(1,1788)=5.232, p=.022)$ and a significant Group-by-CS interaction $(F(2,1788)=4.859, p=.008)$, Figure 2A. Contrary to our hypothesis 1$)$, the Fasting 
Group showed lower probability to avoid than the Re-feeding Group, specifically during the CS- relatively to the $\mathrm{CS}_{\text {avoidable }}\left(\exp -\beta=.320 \pm .398, t=-2.862_{(1788)}, p=.004,95 \%\right.$ CIs [Lower: .147; Upper: .699]) and during the CS- relatively to the $\mathrm{CS}+_{\text {unavoidable }}\left(\exp -\beta=.482 \pm .304, t=-2.405_{(1788)}, p=.016,95 \%\right.$ CIs [Lower: .265 ; Upper: .874]). Results from Post hoc GLMMs run separately for each CS (split: 'CS', factor 3) indeed indicated a significant Group by Trial interaction only for the CS-, driven by a lower probability to press the button over the trials in the Fasting group compared to the Re-feeding group $\left(\exp -\beta=.741 \pm .107, t=-2.798_{(596)}, p=.005\right.$, 95\% CIs [Lower: .600; Upper: .914]). In this model, there was also a significant main effect of Group, with the Fasting group showing a lower probability to press the button during the CS- compared to the Re-feeding group $\left(\exp -\beta=.110 \pm .984, t=-2.246_{(596)}, p=.025,95 \%\right.$ CIs [Lower: .016; Upper: .758]), although this result did not survive the correction for multiple comparisons applied after the split.

Relief-pleasantness ratings: The 2 (Group: Fasting, Re-feeding) by 2 (CS: CS+ $+_{\text {avoidable, }}$ CS-) by Trial (continuous variable) GLMM analysis revealed a Group by CS interaction $(F(1,1170)=20.115, p<.001)$, with the Fasting Group unexpectedly showing lower relief for the CS- relatively to the $\mathrm{CS}+{ }_{\text {avoidable }}$ than the refeeding group ( $\exp -\beta=$ $2.876 \pm .235, t=-4.485_{(1170)}, p<.001,95 \%$ CIs [Lower: 1.812 ; Upper: 4.565$\left.]\right)$, Figure 2B. To further explore the interaction, we ran two separate GLMMs for the two CS (split: 'CS', factor 2). Results from these analyses showed the a trend for the Main effect of Group only for the CS-. Specifically, during the CS-, the Fasting group showed lower probability to report high relief compared to the Re-feeding group ( $\exp -\beta=.259 \pm .761, t=-1.774_{(593)}, p=.077$, 95\% CIs [Lower: .058; Upper: 1.156]).

Anticipatory SCR: The 2 (Group: Fasting and Re-feeding Group) by 3 (CS: CS-, CS+ $+_{\text {avoidable, }}$ CS $+_{\text {unavoidable }}$ ) by Trial (continuous variable) analysis of the lognormal component revealed an unexpected Group by CS interaction $(F(2,782.034)=3.333, p=.036)$. Specifically, we found a higher SCR during CS anticipation in the Re-feeding group than the Fasting group during the CS+ $+_{\text {avoidable }}$ compared to the CS- $(\beta=-.248 \pm .097, t$ :

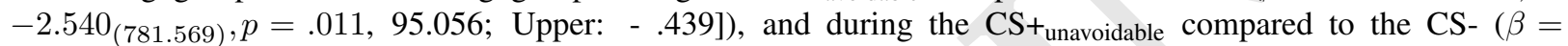
$.202 \pm .099, t=2.023_{(782.689)}, p=.043,95 \%$ CIs [Lower: .006; Upper: .397]), Figure 2C. Accordingly, the results from Post hoc LMM analyses ran separately for each CS (split: 'CS', factor 3) showed a lower SCR in the Fasting group than in the Re-feeding Group for both the $\mathrm{CS}+_{\text {avoidable }}\left(\beta=-.299 \pm .125, t_{(43.158)}=-2.395, p=.021,95 \%\right.$ CIs [Lower: - .551; Upper: - .047], $\mathrm{d}=.491)$ and the $\mathrm{CS}+_{\text {unavoidable }}\left(\beta=-.237 \pm .108, t=-2.192_{(53.356)}, p=.033\right.$, 95\% CIs [Lower: - .454; Upper: - .020], $\mathrm{d}=.210$ ), although these results did not survive the Bonferroni correction. Finally, the results from the logit component showed no significant main effect of Group $(p>.050)$, see Table S5 in Supplementary Material.

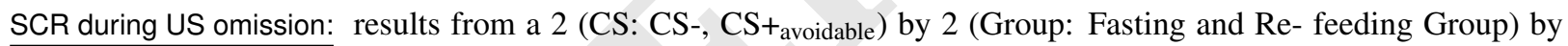
Trial (continuous variable) LMM analysis indicated no significant Group- related effects, see Table S6 in Supplementary Material.

Reaction times: no significant differences in avoidance reaction time were found between the groups for any of the three CSs (see Supplementary Material).

Collectively, these results indicate that fasting reduced unnecessary avoidance and relief pleasantness after safe signals during avoidance learning. Additionally, fasting reduced the magnitude of the physiological anticipatory response to dangerous stimuli.

\section{Extinction learning: overnight fasting decreases relief pleasantness (hypothesis 2).}

Retrospective US-expectancy ratings: In contrast to our hypothesis, the 2 (Group: Fasting, Re-feeding) by 2 (CS) by 2 (Time: first vs last presentation) LMM did not yield any significant Group-related effects.

Anticipatory SCR: Differently from what we hypothesized, the 2 (Group: Fasting, Re- feeding) by 2 (CS) by Trial (continuous variable) LMM analysis displayed no Group-related effects.

Relief pleasantness ratings: The 2 (Group: Fasting and Re-feeding Group) by 2 (CS) by Trial (continuous variable) GLMM analysis revealed a unexpected significant Group by Trial interaction $(F(1,789)=11.430, p=.001)$, with lower probability to report an increase in the relief ratings over trials in the Fasting Group compared to the Re-feeding Group, for both CSs $\left(\exp -\beta=.802 \pm .097, t=-2.270_{(789)}, p=.023,95 \%\right.$ CIs [Lower: .663; Upper: .971]). Additionally, there was a significant unexpected Group by CS interaction $(\mathrm{F}(1,789)=11.525, \mathrm{p}=.001)$, where 

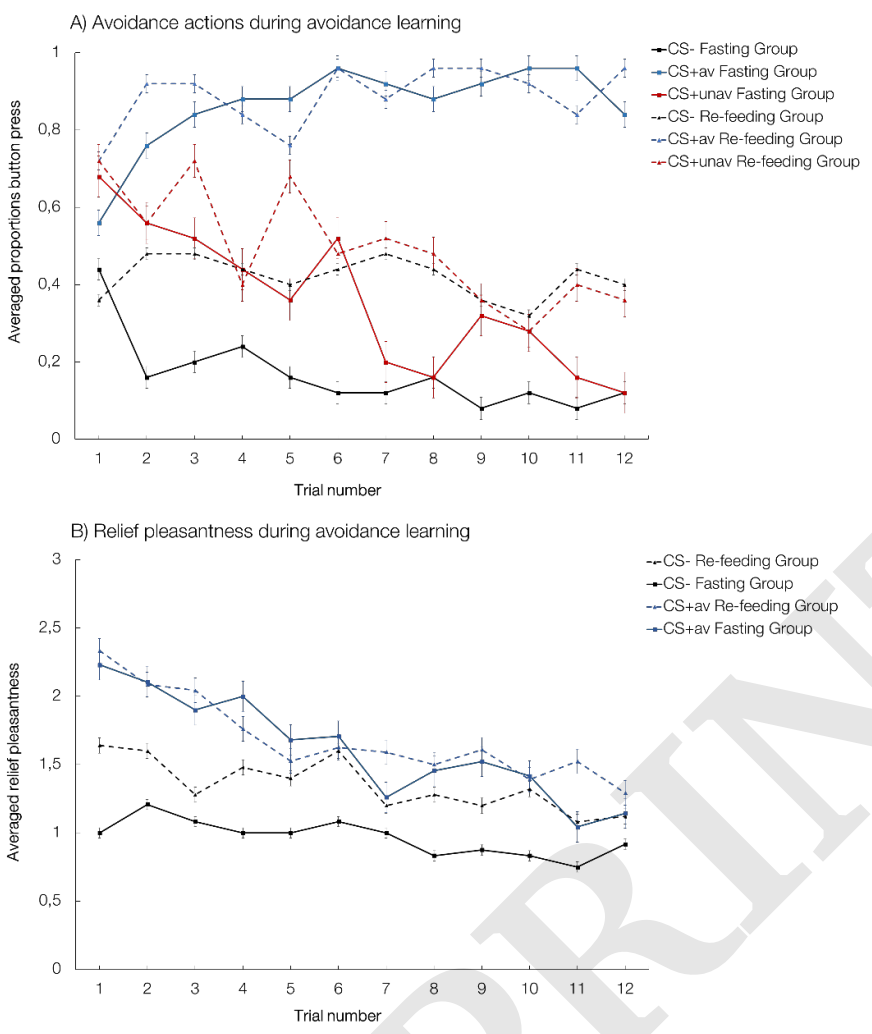

--CS- Re-feeding Group $\rightarrow$ CS- Fasting Group --CS+av Re-feeding Group - CS+av Fasting Group

C) Magnitude of the SCR during US anticipation (Lognormal component)

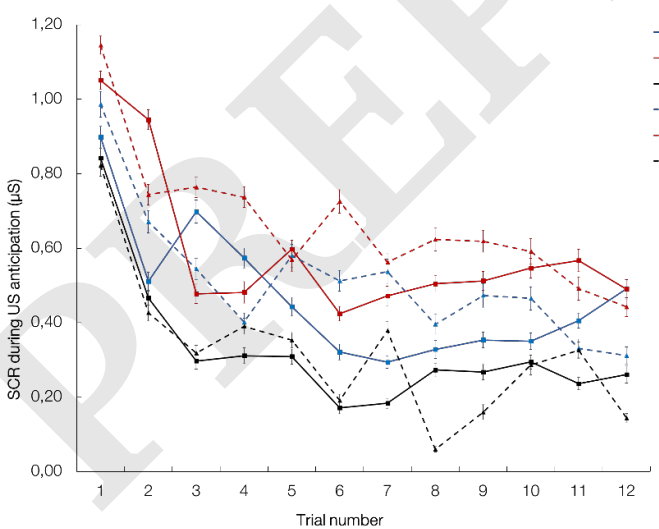

- CS+av Fasting Group - CS+unav Fasting Group -CS- Fasting Group - CS+av Re-feeding Group -CSt+unav Re-feeding Group -CS- Re-feeding Group

Figure 2. Results from the Avoidance learning phase across the Fasting and the Re-feeding Group. The average button presses for the CS-, CS $+_{\text {avoidable }}$ and $C S+_{\text {unavoidable }}(\mathrm{A})$ : compared to the Re-feeding group, the Fasting group showed a reduction in the

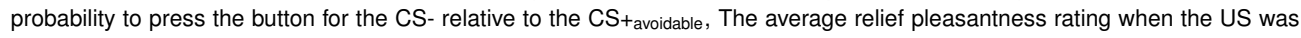
successfully omitted ( $\left.\mathrm{CS}+_{\text {avoidable }}\right)$ and during the signaled absence of threat (CS-) (B): compared to the Re- feeding group, the

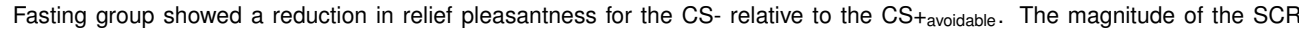
during US anticipation for both the Fasting and the Re-feeding Group $(\mathrm{C})$ : compared to the Refeeding Group, the Fasting Group showed a lower SCR during US anticipation. Error bars represent the standard error of the mean. 


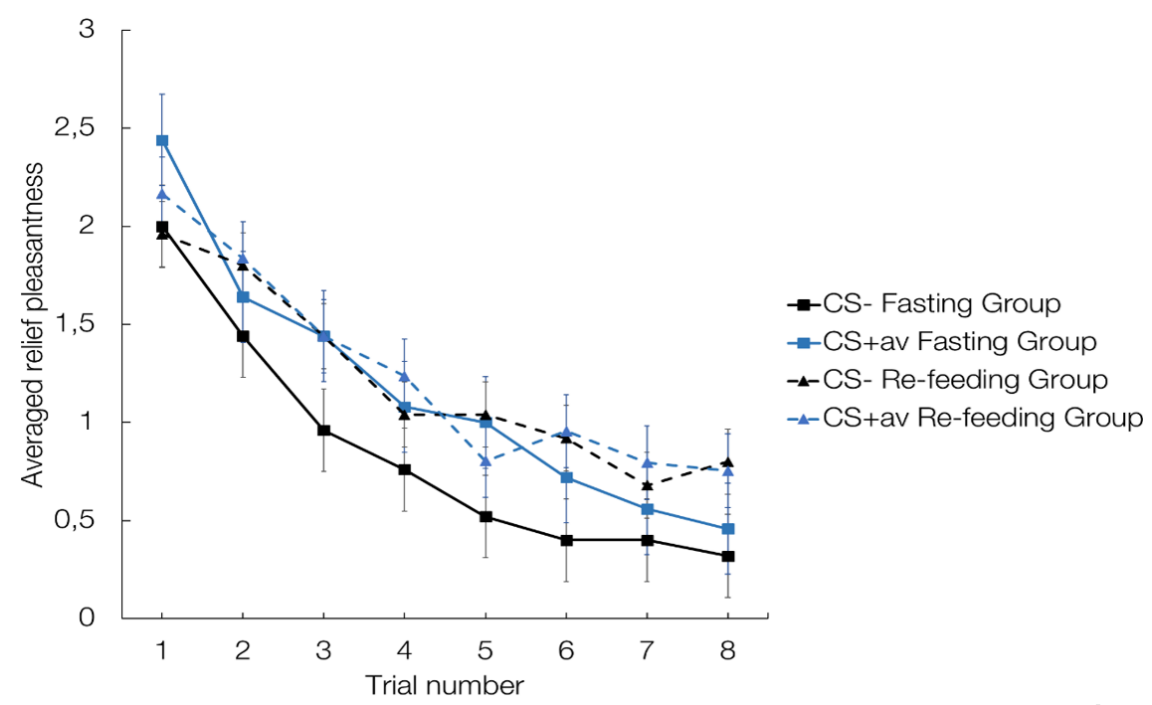

Figure 3. The results from relief pleasantness ratings (averages) during the fear extinction phase for the Fasting and the Refeeding Group. Error bars represent the standard error of the mean. Compared to the Re-feeding group, the Fasting group showed a significant decrease in relief pleasantness for the CS- relative to the CS+avoidable.

the Fasting group reported a lower probability of high ratings for the CS- relatively to the $\mathrm{CS}+{ }_{\text {avoidable }}(\exp -\beta=$ $.354 \pm .306, t=-3.395_{(789)}, p=.001,95 \%$ CIs [Lower: .194; Upper: .645]), Figure 3. Results from Post hoc GLMMs ran separately for the two CSs (split: 'CS', factor 2) showed a trend for the main effect of Group only for the CS-. Specifically, compared to the Re-feeding group, the Fasting group showed lower probability to report high relief ratings for the $\mathrm{CS}-\left(\exp -\beta=.258 \pm .680, t=-1.989_{(394)}, p=.047,95 \%\right.$ CIs [Lower: .068; Upper: .984]). For SCR during US omission: no significant Group-related effects were found.

To sum up, overnight fasting reduced the relief pleasantness experienced after safe rather than dangerous stimuli during extinction learning.

\section{The mediational role of relief: overnight fasting optimizes avoidance learning by decreasing differential relief (hypothesis 3 ).}

We examined whether the observed effect of overnight fasting on avoidance frequency in the avoidance learning phase was mediated by changes in relief, hypothesis 3). Given the small sample size we did not implement a structural equation-based cross-lagged panel model on trial-by-trial data, but rather a simple mediation analysis based on averaged-based measures of relief and avoidance. For the calculation of the relief (Mediator, M) and avoidance (dependent variable, Y) index, we used relief and avoidance to CS $+_{\text {avoidable }}$ compared to CS- (differential relief). We excluded the last relief rating of the avoidance phase since this was not temporally followed by any further trial, and so, it was not predictive of any further avoidance action. Hence, the averaged relief pleasantness for the first 7 trials of the CS- was subtracted from the averaged relief pleasantness of the first 7 trials of the CS+ avoidable and used as mediator (M). Similarly, for the calculation of the proportion of differential avoidance, we did not include the first avoidance trial since this was not preceded by any relief rating. Hence, for this index, we calculate the proportion of

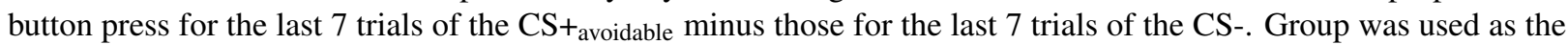
independent variable $(\mathrm{X})$. Next, a mediation analysis was run with PROCESS 3.5 in SPSS.

The results showed that Group was negatively associated with differential avoidance, $\mathrm{X} \rightarrow \mathrm{Y}$ (direct path c: $\beta=$ $\left.-.273 \pm .115, t_{(1,48)}=-2.380, p=.021\right)$, in line with mixed model results reported earlier. Additionally, differential relief was positively associated with differential avoidance $\left(\mathrm{M} \rightarrow \mathrm{Y}\right.$, path $\mathrm{b}: \beta=.323 \pm .110, t_{(2,47)}=2.916, p=$ $.005)$, while group was negatively associated with differential relief $\left(\mathrm{X} \rightarrow \mathrm{M}\right.$, path a : $\beta=-.332 \pm .139, t_{(1,48)}=$ $-2.396, p=.021)$. Most importantly, path c changed from significant to non-significant when the indirect path was included in the model ( $\left.c^{\prime}: \beta=-.165 \pm .113, t_{(2,47)}=-1.466, p=.149\right)$, see Figure 4 . Results thus confirmed the full mediating role of differential relief in the relation between Group and differential avoidance $(\beta=.323$, CI $=$ .102 to .633). To sum up, differential relief pleasantness fully mediated the effect of Group on differential avoidance, indicating that overnight fasting optimizes instrumental learning by reducing the positive effects of relief pleasantness on avoidance. 


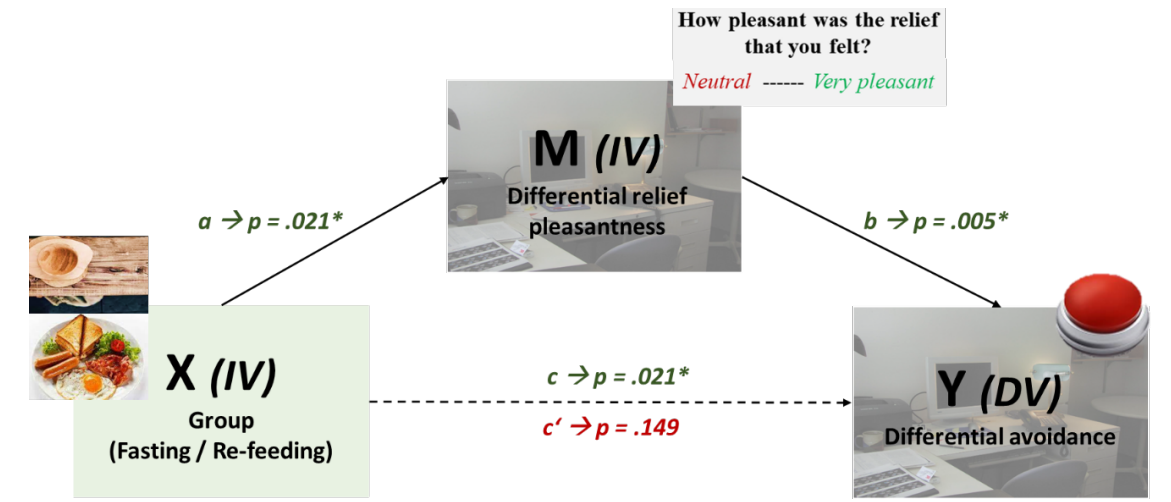

Figure 4. Results from the mediation analysis for hypothesis 3. The Groups (Fasting and Re-feeding) and the mediator (the differential relief pleasantness) were used as Independent Variables [IV] to predict the level of the differential avoidance (dependent variable [DV]) during the avoidance learning phase. Higher differential relief pleasantness fully mediated the effect of Fasting (vs re-feeding) on reducing differential avoidance actions.

\section{Test phases: overnight fasting reduces persistent avoidance in the extinction context (hypothesis $4 a$ and $4 b)$.}

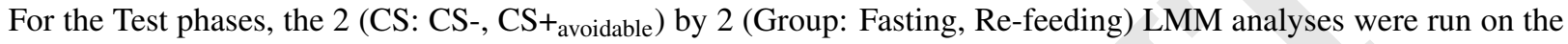
average of the avoidance actions recorded for each level of the CS. This choice was made in light of the small number of trials used in the test phases [from thirty-six (Avoidance learning phase) to eight trials within each Test phase].

Test 1 showed the absence of a significant Group by CS interaction $(F(1,48)=0.18, p=.849)$. There was, however, a main effect Group $(F(1,48)=4.809, p=.033)$ with the Fasting Group avoiding less than the Re-feeding Group $\left(\beta=-.195 \pm .089, t=-2.193_{(48)}, d=.461\right)$. This result was in the reverse direction from what we described in hypothesis $4 \mathrm{a}$ ). We investigated whether the observed effect of overnight fasting on avoidance frequency in the test phase was mediated by changes in relief during the previous avoidance learning phase, hypothesis $4 \mathrm{~b}$ ). To address this last hypothesis, we e run a sequential mediation analysis that included as a first Mediator (M1) the differential relief pleasantness (same index used for the previous mediation), and, as a second Mediator (M2), the difference in proportion of differential avoidance (same index used for the previous mediation). The two Mediators were taken from the avoidance learning phase of the task. Group was used as independent variable (X), while the average of button press recorded during Test 1 was now used as dependent variable (Y). The results showed that Group was positively associated with a return in avoidance at Test $1, \mathrm{X} \rightarrow \mathrm{Y}$ (direct path $\mathrm{c}: \beta=.195 \pm .089, t_{(1,48)}=2.193, p=.033$ ), in line with mixed model results reported above. As already found for hypothesis 3 , Group was negatively associated with differential relief $\left(\mathrm{X} \rightarrow \mathrm{M} 1\right.$, path a1 : $\left.\beta=-.332 \pm .139, t_{(1,48)}=-2.396, p=.021\right)$, and differential relief was positively associated with differential avoidance $\left(\mathrm{M} 1 \rightarrow \mathrm{M} 2\right.$, path $\left.\mathrm{d} 21: \beta=.323 \pm .110, t_{(2,47)}=2.916, p=.005\right)$. Furthermore, differential avoidance was negatively associated with a return in avoidance at Test 1 (M2 $\rightarrow$ Y, path b2: $\left.\beta=-.385 \pm .103, t_{(3,46)}=-3.743, p=.001\right)$. Additionally, no direct association was found neither with Group and differential avoidance $\mathrm{X} \rightarrow \mathrm{M} 2$, path $\left.\mathrm{a} 2: \beta=-.165 \pm .113, t_{(3,46)}=-1.466, p=.149\right)$, or with differential relief and a return in avoidance at Test $1(\mathrm{M} 1 \rightarrow \mathrm{Y}$, path b1: $\beta=-.084 \pm .085, t(3,46)=-.987, p=.329)$. Most importantly, path c changed from significant to non-significant exclusively when the serial indirect path was included in the model (c': $\beta=.062 \pm .081, t_{(3,46)}=.762, p=.450$ ), see Figure 5. Opposite to what we expected (hypothesis $4 \mathrm{~b}$ ), these results indicate that the effects of overnight fasting on the reduction of avoidance during Test 1 were dependent on the previous group differences in the button clicks, which were mediated by the unexpected differences in (reduced) relief ratings found during the avoidance learning phase $(\beta=.041, \mathrm{CI}=.003$ to .136$)$.

Test 2 showed similar numerical results as Test 1 , but the Group main effect $(F(1,48)=1.940, p=.170)$ and the Group by $\mathrm{CS}$ interaction $(\mathrm{F}(1,47)=.711, \mathrm{p}=.403)$ were not significant, Figure 6 . 


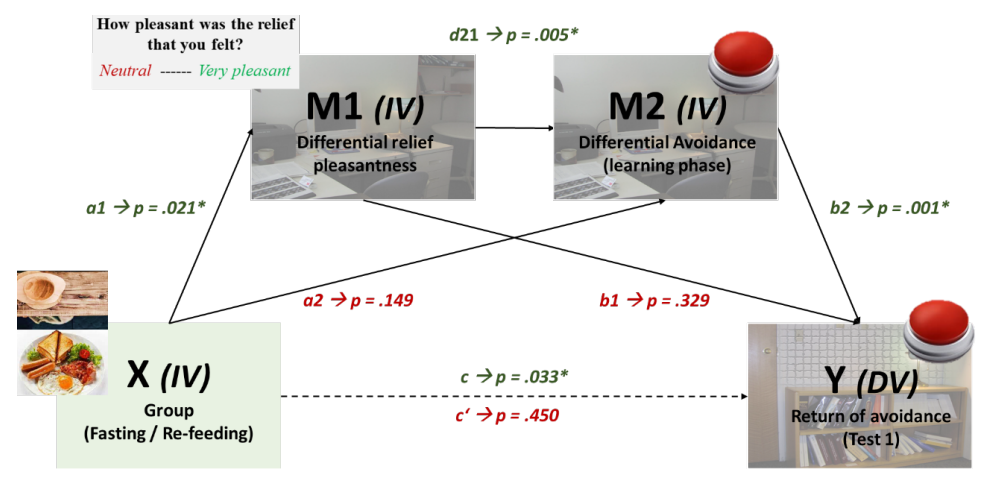

Figure 5. Results from the serial mediation analysis for hypothesis 4b. The Groups (Fasting and Re-feeding) and the mediators (the differential relief pleasantness and the differential avoidance) were used as Independent Variables [IV] to predict the frequency of button press at Test 1 (dependent variable [DV]). Higher differential relief pleasantness and differential avoidance during learning fully mediated the effect of Fasting (vs re-feeding) on reducing a return of avoidance after fear extinction.
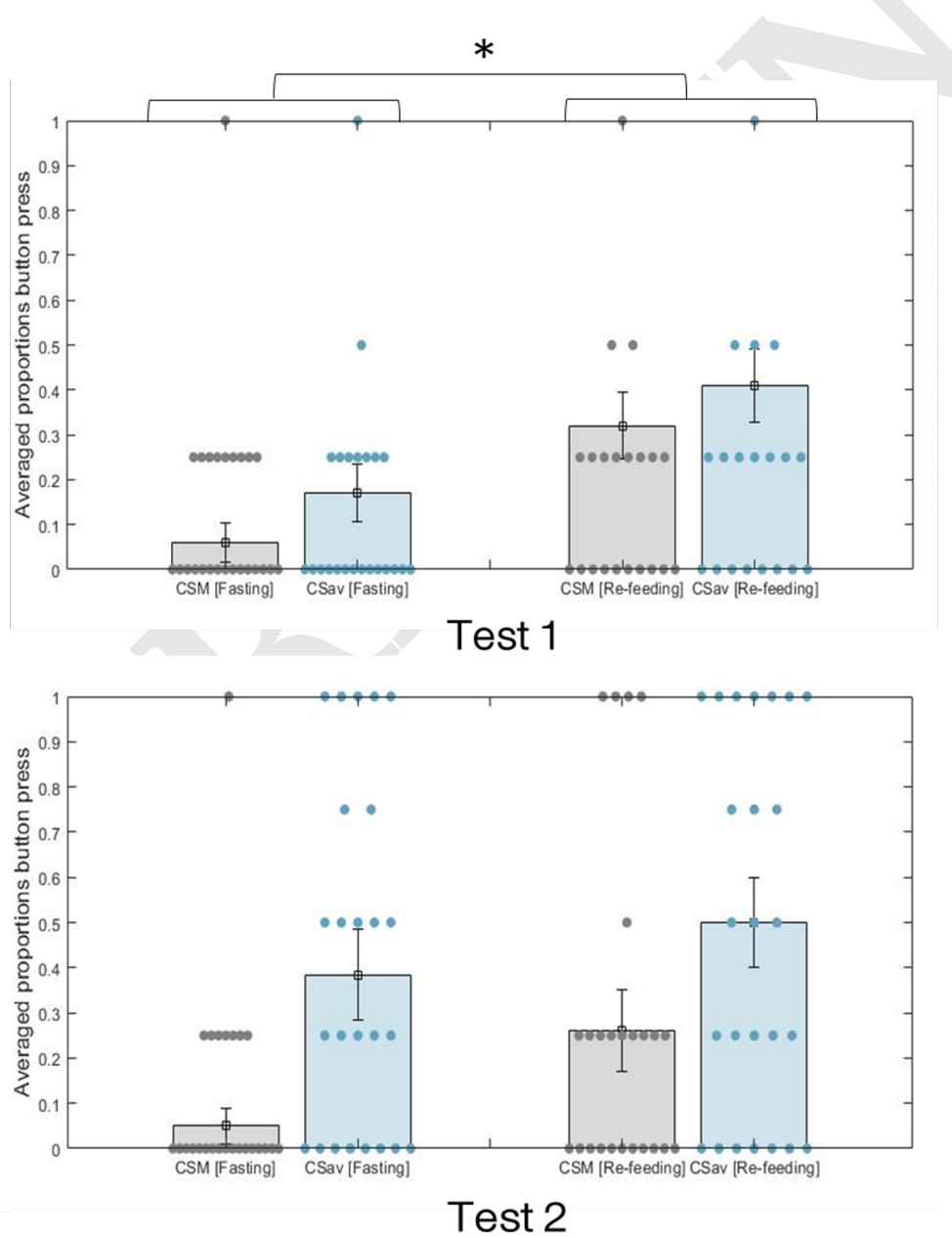

Figure 6. Proportion of avoidance actions (button press) across participants for each CS, during Test 1 (upper panel) and Test 2 (bottom panel), for both the Fasting and the Re-feeding group. Compared to the Re-feeding group the Fasting group generally showed less button press at Test 1. A similar but non-significant trend was found on Test 2. 


\section{Discussion}

Our study examined the effects of overnight fasting on avoidance learning, fear extinction, and relief pleasantness. We found that overnight fasting 1) optimized avoidance decisions by selectively reducing unnecessary avoidance actions, and 2) reduced relief pleasantness to signaled threat omissions during extinction learning. Furthermore, the reduction in unnecessary vs necessary avoidance was mediated by a reduction in differential relief pleasantness during avoidance learning (3). The effect of fasting on avoidance learning contributed to a persistent reduction of avoidance after fear extinction (4a); while (4b) the reduction in avoidance at test 1 in the fasting group was mediated by the reduction in differential relief pleasantness during avoidance learning. Avoidance is a form of safety learning that depends on dopaminergic signaling. Based on the evidence that fasting enhances safety learning (fear extinction) and sensitizes the dopaminergic system, we hypothesized that overnight fasting would increase avoidance actions during learning (and persistence of avoidance after fear extinction) by increasing the reinforcing effects of relief induced by successful threat omission (which serves as reward). Contrary to what we hypothesized, overnight fasting decreased the pleasantness of relief for passive omissions after the safe CS-, but not for active omissions

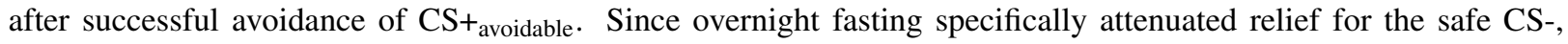
we investigated whether the reduction in differential relief mediated the reduction in differential avoidance learning, which was indeed the case. Additionally, such fasting-related changes in learning and relief led to reduced persistent avoidance during test, after fear was extinguished, specifically within the safe context.

These findings indicate that fasting acts as a promotor of adaptive behaviors. Theoretically, these results are consistent with principles of Maslow's hierarchy of needs[19]. These principles stipulate that human needs are hierarchically organized, with the physiological need (e.g., food) and the need for safety (protection from threat) as the most important ones for survival. It is regularly observed across species that these needs act as primary rewards that motivate goal-directed behaviors. However, these two needs often compete with each other. Specifically, hungry animals need to reduce their need for safety in order to face (and not avoid) the threat to obtain food. Accordingly, here, overnight fasting might represent a homeostatic state that motivates the subjects to avoid less (and selectively) by simultaneously increasing the reward value for food and decreasing the need for safety (in the present task, when safety is guaranteed, CS-) as well as the general physiological arousal during stimulus presentation. It is possible indeed that the Fasting Group chooses to press the button selectively when effective ( $\left.\mathrm{CS}++_{\text {avoidable }}\right)$, given a reduction on safety needs.

We did not observe any effects of fasting on extinction learning in terms of retrospective expectancies or online SCR. This result is partially in contrast with what has been found in animal studies[13, 40], but is in line with the results from the study of Le Shi et al., 2018[35], who reported fasting effects on the consolidation of fear extinction, but not on its acquisition. Since our protocol was focused on avoidance learning and two tests within the same day, we were, however, not able to investigate the effects of overnight fasting on the consolidation of fear extinction one day after acquisition. Nevertheless, we found evidence for a decrease in relief pleasantness for the CS- after overnight fasting during extinction learning. This suggests that overnight fasting might optimize safety learning by affecting reward- related indexes (relief) rather than fear-related indexes (e.g., US expectancies and/or anticipatory SCR). Notably, we recently proposed that relief levels might be used as an index of safety learning (in addition to indices of fear) in individuals that participate in extinction/exposure training[32, 43].

We propose that future studies should investigate the biological mechanisms underlying the effects of overnight fasting on avoidance and relief pleasantness. Central attention should be given to gut-brain signaling mediators following acute fasting[17] such as ghrelin; to central neurotransmitters with regulatory effects on the mesolimbic system, like dopamine; and to their interaction. Across species, the effects of fasting on fear are mediated by ghrelin, a hormone produced in the gastrointestinal tract that rises during fasting. Its receptors are strongly expressed in the VTA and NAcc[21], brain regions governing error-based learning (i.e. fear extinction[14, 18, 31], and reward learning[4]). Ghrelin activates dopaminergic neurons in the VTA, which in turn increase dopaminergic turnover in the NAcc[1]. For this reason, it would be interesting to investigate if overnight fasting reduces unnecessary/ineffective avoidance by modulating VTA-NAcc brain activation during US omissions. The mechanisms of food deprivation-driven changes in relief-value should also be further characterized at a higher cortical level. In this respect, overnight fasting might impact orbitofrontal cortex responses to US omissions, a region representing reward value[29, 33, 34]; these changes might then mediate the effects of fasting on avoidance learning, which we demonstrated here. Finally, it would be clinically relevant to investigate fasting effects in individuals with excessive fear and avoidance behaviors. Indeed, we recently proposed that in anxious individuals the affective value of the relief might be increased. As a result of this increased pleasantness of relief, the individual will perform that same avoidance action in the future more frequently, even if fear is reduced. Based on the present results, it might be hypothesized that a reduction in relief pleasantness 
following fasting might support safety learning during exposure therapies, which may in turn reduce fear/avoidance.

Two notes of caution regarding our results are warranted. First, since only women were included, future studies should extend these results by including men. Additionally, we consider it unlikely that the effects we found were dependent on a drop in attention levels following food deprivation, since no significant group differences in RTs were found for each of the three CSs. However, replication studies should consider potential effects of fasting on other dopamine-based cognitive skills, like working memory capacity[11], which might, in turn, affect the performance of the participants.

To conclude, we demonstrated that overnight fasting contributes to an optimal avoidance profile by reducing unnecessary avoidance and relief during safety. Future studies should investigate the neural underpinnings of these effects and whether overnight fasting produces a reduction in excessive avoidance within a clinical setting. Finally, we recommend future behavioral-neuroimaging studies on safety-based learning within the context of fear and avoidance to consider the hunger state of the participants.

\section{Acknowledgements}

BV was supported by a KU Leuven starting grant STG-18-00299, LVO is an associate research professor funded by the KU Leuven Special Research Fund (BOF), TB is supported by Consolidator Grant 648176 of the European Research Council. The founders had no role in study design, data collection and analysis, decision to publish or preparation of the manuscript.

\section{Contributions}

BV, LVO and SP developed the concepts of the study and study design; SP and LN performed the collection of the data; SP performed the data analysis and interpretation under the supervision of BV and LVO; SP drafted the manuscript under the supervision of BV and LVO. All the authors provided revisions, conceptual and statistical feedback, and approved the final version of the manuscript for submission.

\section{Data availability}

Original data are stored and secured within the KU Leuven facilities given ethical reasons. Processed anonymous data are available on the Open Science Framework (OSF), https://osf .io/yqp3x/.

\section{References}

1. A. Abizaid, Z.-W. Liu, Z. B. Andrews, M. Shanabrough, E. Borok, J. D. Elsworth, R. H. Roth, M. W. Sleeman, M. R. Picciotto, M. H. Tschöp, X.-B. Gao, and T. L. Horvath. Ghrelin modulates the activity and synaptic input organization of midbrain dopamine neurons while promoting appetite. The Journal of Clinical Investigation, 116(12):3229-3239, Dec. 2006.

2. American Psychiatric Association. Diagnostic and statistical manual of mental disorders. Autor, Washington, DC, 5th ed. edition, 2013.

3. D. R. Bach, G. Flandin, K. J. Friston, and R. J. Dolan. Time-series analysis for rapid event-related skin conductance responses. Journal of Neuroscience Methods, 184(2):224-234, Nov. 2009.

4. K. C. Berridge, T. E. Robinson, and J. W. Aldridge. Dissecting components of reward: 'liking', 'wanting', and learning. Current Opinion in Pharmacology, 9(1):65-73, Feb. 2009.

5. R. C. Bolles. Species-specific defense reactions and avoidance learning. Psychological Review, 77(1):32-48, 1970. Place: US Publisher: American Psychological Association.

6. S. Y. Branch, R. B. Goertz, A. L. Sharpe, J. Pierce, S. Roy, D. Ko, C. A. Paladini, and M. J. Beckstead. Food Restriction Increases Glutamate Receptor-Mediated Burst Firing of Dopamine Neurons. Journal of Neuroscience, 33(34):13861-13872, Aug. 2013.

7. C. Bravo-Rivera, C. Roman-Ortiz, M. Montesinos-Cartagena, and G. J. Quirk. Persistent active avoidance correlates with activity in prelimbic cortex and ventral striatum. Frontiers in Behavioral Neuroscience, 9, July 2015.

8. M. Brysbaert and M. Stevens. Power Analysis and Effect Size in Mixed Effects Models: A Tutorial. Journal of Cognition, 1(1):9, Jan. 2018. Number: 1 Publisher: Ubiquity Press.

9. R. M. Cassidy and Q. Tong. Hunger and Satiety Gauge Reward Sensitivity. Frontiers in Endocrinology, 8, 2017. Publisher: Frontiers.

10. J. J. Cone, J. E. McCutcheon, and M. F. Roitman. Ghrelin acts as an interface between physiological state and phasic dopamine signaling. The Journal of Neuroscience: The Official Journal of the Society for Neuroscience, 34(14):4905-4913, Apr. 2014. 
11. R. Cools and M. D'Esposito. Inverted-U shaped dopamine actions on human working memory and cognitive control. Biological psychiatry, 69(12):e113-e125, June 2011.

12. A. P. Goldstone, C. G. Prechtl, S. Scholtz, A. D. Miras, N. Chhina, G. Durighel, S. S. Deliran, C. Beckmann, M. A. Ghatei, D. R. Ashby, A. D. Waldman, B. D. Gaylinn, M. O. Thorner, G. S. Frost, S. R. Bloom, and J. D. Bell. Ghrelin mimics fasting to enhance human hedonic, orbitofrontal cortex, and hippocampal responses to food. The American Journal of Clinical Nutrition, 99(6):1319-1330, June 2014.

13. C.-C. Huang, D. Chou, C.-M. Yeh, and K.-S. Hsu. Acute food deprivation enhances fear extinction but inhibits long-term depression in the lateral amygdala via ghrelin signaling. Neuropharmacology, 101:36-45, Feb. 2016.

14. R. Kalisch, A. M. V. Gerlicher, and S. Duvarci. A Dopaminergic Basis for Fear Extinction. Trends in Cognitive Sciences, 23(4):274-277, Apr. 2019.

15. A.-M. Krypotos, M. Effting, M. Kindt, and T. Beckers. Avoidance learning: a review of theoretical models and recent developments. Frontiers in Behavioral Neuroscience, 9, 2015. Publisher: Frontiers.

16. S. Leknes, M. Lee, C. Berna, J. Andersson, and I. Tracey. Relief as a reward: hedonic and neural responses to safety from pain. PloS One, 6(4):e17870, Apr. 2011.

17. L. Li, Y. Su, F. Li, Y. Wang, Z. Ma, Z. Li, and J. Su. The effects of daily fasting hours on shaping gut microbiota in mice. BMC Microbiology, 20(1):65, Mar. 2020.

18. R. Luo, A. Uematsu, A. Weitemier, L. Aquili, J. Koivumaa, T. J. McHugh, and J. P. Johansen. A dopaminergic switch for fear to safety transitions. Nature Communications, 9(1):2483, 2018.

19. A. H. Maslow. A Dynamic Theory of Human Motivation. In Understanding human motivation, pages 26-47. Howard Allen Publishers, Cleveland, OH, US, 1958.

20. C. E. Mcculloch and J. M. Neuhaus. Generalized Linear Mixed Models. In Wiley StatsRef: Statistics Reference Online. American Cancer Society, 2014.

21. J. R. W. Menzies, K. P. Skibicka, G. Leng, and S. L. Dickson. Ghrelin, Reward and Motivation. The Ghrelin System, 25:101-111, 2013.

22. J. S. Morris and R. J. Dolan. Involvement of human amygdala and orbitofrontal cortex in hunger-enhanced memory for food stimuli. The Journal of Neuroscience: The Official Journal of the Society for Neuroscience, 21(14):5304-5310, July 2001.

23. O. H. Mowrer. Learning theory and behavior. Learning theory and behavior. John Wiley \& Sons Inc, Hoboken, NJ, US, 1960.

24. E. B. Oleson and J. F. Cheer. On the role of subsecond dopamine release in conditioned avoidance. Frontiers in Neuroscience, 7 , 2013. Publisher: Frontiers.

25. D. E. Pankevich, S. L. Teegarden, A. D. Hedin, C. L. Jensen, and T. L. Bale. Caloric Restriction Experience Reprograms Stress and Orexigenic Pathways and Promotes Binge Eating. Journal of Neuroscience, 30(48):16399-16407, Dec. 2010.

26. S. Papalini, M. Ashoori, J. Zaman, T. Beckers, and B. Vervliet. The role of context in persistent avoidance and the predictive value of relief. Behaviour Research and Therapy, 138:103816, Mar. 2021.

27. S. Papalini, T. Beckers, and B. Vervliet. Dopamine: from prediction error to psychotherapy. Translational Psychiatry, 10(1):164, 2020.

28. K. J. Pultorak, S. A. Schelp, D. P. Isaacs, G. Krzystyniak, and E. B. Oleson. A Transient Dopamine Signal Represents Avoidance Value and Causally Influences the Demand to Avoid. eNeuro, 5(2), May 2018.

29. E. T. Rolls, W. Cheng, and J. Feng. The orbitofrontal cortex: reward, emotion and depression. Brain Communications, 2(2):fcaa196, Nov. 2020.

30. A. G. Roseberry. Acute fasting increases somatodendritic dopamine release in the ventral tegmental area. Journal of Neurophysiology, 114(2):1072-1082, Aug. 2015.

31. X. I. Salinas-Hernández, P. Vogel, S. Betz, R. Kalisch, T. Sigurdsson, and S. Duvarci. Dopamine neurons drive fear extinction learning by signaling the omission of expected aversive outcomes. eLife, 7, 2018.

32. C. San Martín, B. Jacobs, and B. Vervliet. Further characterization of relief dynamics in the conditioning and generalization of avoidance: Effects of distress tolerance and intolerance of uncertainty. Behaviour Research and Therapy, 124:103526, Jan. 2020.

33. G. Sescousse, J. Redouté, and J.-C. Dreher. The Architecture of Reward Value Coding in the Human Orbitofrontal Cortex. The Journal of Neuroscience, 30(39):13095-13104, Sept. 2010.

34. T. Setogawa, T. Mizuhiki, N. Matsumoto, F. Akizawa, R. Kuboki, B. J. Richmond, and M. Shidara. Neurons in the monkey orbitofrontal cortex mediate reward value computation and decision-making. Communications Biology, 2(1):1-9, Apr. 2019.

35. L. Shi, J. Deng, S. Chen, J. Que, Y. Sun, Z. Wang, X. Guo, Y. Han, Y. Zhou, X. Zhang, W. Xie, X. Lin, J. Shi, and L. Lu. Fasting enhances extinction retention and prevents the return of fear in humans. Translational Psychiatry, 8, Oct. 2018.

36. J. Skrynka and B. T. Vincent. Hunger increases delay discounting of food and non-food rewards. Psychonomic Bulletin \& Review, 26(5):1729-1737, Oct. 2019.

37. A. Spruyt, J. Clarysse, D. Vansteenwegen, F. Baeyens, and D. Hermans. Affect 4.0. Experimental Psychology, 57(1):36-45, Oct. 2010. Publisher: Hogrefe Publishing.

38. E. Stice, K. Burger, and S. Yokum. Caloric Deprivation Increases Responsivity of Attention and Reward Brain Regions to Intake, Anticipated Intake, and Images of Palatable Foods. Neurolmage, 67:322-330, Feb. 2013.

39. J. A. Tooze, G. K. Grunwald, and R. H. Jones. Analysis of repeated measures data with clumping at zero. Statistical Methods in Medical Research, 11(4):341-355, Aug. 2002.

40. D. Verma, J. Wood, G. Lach, H. Herzog, G. Sperk, and R. Tasan. Hunger Promotes Fear Extinction by Activation of an Amygdala Microcircuit. Neuropsychopharmacology: Official Publication of the American College of Neuropsychopharmacology, 41(2):431-439, Jan. 2016.

41. B. Vervliet, M. G. Craske, and D. Hermans. Fear extinction and relapse: state of the art. Annual Review of Clinical Psychology, 9:215-248, 2013

42. B. Vervliet and E. Indekeu. Low-Cost Avoidance Behaviors are Resistant to Fear Extinction in Humans. Frontiers in Behavioral Neuroscience, 9, Dec. 2015.

43. B. Vervliet, I. Lange, and M. R. Milad. Temporal dynamics of relief in avoidance conditioning and fear extinction: Experimental validation and clinical relevance. Behaviour Research and Therapy, 96:66-78, Sept. 2017.

44. X. J. Wei, B. Sun, K. Chen, B. Lv, X. Luo, and J. Q. Yan. Ghrelin signaling in the ventral tegmental area mediates both reward-based feeding and fasting-induced hyperphagia on high-fat diet. Neuroscience, 300:53-62, Aug. 2015.

45. A. L. Willems and B. Vervliet. When nothing matters: Assessing markers of expectancy violation during omissions of threat. Behaviour 
Research and Therapy, 136:103764, Jan. 2021.

46. X. Zhang, A. J. Melrose, O. De Santis, S. Luo, K. A. Page, E. Hsu, and J. R. Monterosso. Fasting may increase incentive signaling for nonfood rewards. Nutrition Research, 77:43-53, May 2020. 